\title{
Note on some representations of general solutions to homogeneous linear difference equations
}

\author{
Stevo Stević ${ }^{1,2,3^{*}}$, Bratislav Iričanin ${ }^{4,5}$, Witold Kosmala ${ }^{6}$ and Zdeněk Šmarda ${ }^{3}$
}

\footnotetext{
"Correspondence: sstevic@ptt.rs ${ }^{1}$ Mathematical Institute of the Serbian Academy of Sciences, Knez Mihailova 36/III, 11000 Beograd, Serbia

${ }^{2}$ Department of Medical Research, China Medical University Hospital, China Medical University, Taichung 40402, Taiwan, Republic of China Full list of author information is available at the end of the article
}

\begin{abstract}
It is known that every solution to the second-order difference equation $x_{n}=x_{n-1}+x_{n-2}=0, n \geq 2$, can be written in the following form $x_{n}=x_{0} f_{n-1}+x_{1} f_{n}$, where $f_{n}$ is the Fibonacci sequence. Here we find all the homogeneous linear difference equations with constant coefficients of any order whose general solution have a representation of a related form. We also present an interesting elementary procedure for finding a representation of general solution to any homogeneous linear difference equation with constant coefficients in terms of the coefficients of the equation, initial values, and an extension of the Fibonacci sequence. This is done for the case when all the roots of the characteristic polynomial associated with the equation are mutually different, and then it is shown that such obtained representation also holds in other cases. It is also shown that during application of the procedure the extension of the Fibonacci sequence appears naturally.
\end{abstract}

MSC: $39 A 10$

Keywords: Homogeneous linear difference equation with constant coefficients; General solution; Representation of solutions; Fibonacci sequence 
The Fibonacci sequence $\left(f_{n}\right)_{n \geq 0}$ is the solution to equation (1) with $x_{0}=0$ and $x_{1}=1$ (the solution can be explicitly found in D. Bernoulli's paper [4], as well as the method that is usually used in solving homogeneous linear difference equations with constant coefficients nowadays). The sequence has been investigated for several centuries, and there are a lot of properties and relations which are satisfied by the sequence (see, for example, $[1,12,16,37])$. The sequence is usually defined on $\mathbb{N}$ or $\mathbb{N}_{0}$, but it is easy to see that it can be defined on any set of integers of the form $n \geq n_{0}$, where $n_{0} \in \mathbb{Z}$ is fixed, as well as on the whole $\mathbb{Z}$ (see also the end of this section).

An interesting fact related to the Fibonacci sequence is that every solution to equation (1) can be written in the following form:

$$
x_{n}=x_{0} f_{n-1}+x_{1} f_{n}, \quad n \in \mathbb{N}_{0} .
$$

There are several ways to prove (2). One of the ways is by using known theory. It is well known that general solution to the equation

$$
L_{k, \vec{a}}\left(x_{n}\right):=x_{n}-a_{1} x_{n-1}-\cdots-a_{k} x_{n-k}=0, \quad n \in \mathbb{N}_{0},
$$

where $k \in \mathbb{N}, \vec{a}=\left(a_{1}, \ldots, a_{k}\right), a_{j} \in \mathbb{R}, j=\overline{1, k}$, and $a_{k} \neq 0$, is a $k$-dimensional linear space $[5,10,11,13,14,17,18]$. Let $\operatorname{Sol}\left(L_{k, \vec{a}}\right):=\left\{\left(x_{n}\right)_{n \geq-k}: L_{k, \vec{a}}\left(x_{n}\right) \equiv 0\right\}$ (the space of all solutions to equation (3)). It is also known that there is a natural linear isomorphism $I_{k}$ between the $k$-dimensional real vector space $\mathbb{R}^{k}$ and the space $\operatorname{Sol}\left(L_{k, \vec{a}}\right)$. Namely, if $\left(\vec{e}_{j}\right)_{j=1}^{k} \subset \mathbb{R}^{k}$ is the canonical basis of $\mathbb{R}^{k}$, i.e., $\vec{e}_{j}=(0, \ldots, 0,1,0, \ldots, 0), j=\overline{1, k}$, where 1 is the $j$ th coordinate in the vector, and $\tilde{x}(\vec{v})=\left(x_{n}(\vec{v})\right)_{n \geq-k} \in \operatorname{Sol}\left(L_{k, \vec{a}}\right)$ is the solution corresponding to the vector $\vec{v} \in \mathbb{R}^{k}$ (the set of initial values), then for every $\vec{v}=\sum_{j=1}^{k} v^{j} \vec{e}_{j} \in \mathbb{R}^{k}$ we have

$$
\tilde{x}(\vec{v})=I_{k}(\vec{v})=I_{k}\left(\sum_{j=1}^{k} v^{j} \vec{e}_{j}\right)=\sum_{j=1}^{k} v^{j} I_{k}\left(\vec{e}_{j}\right)=\sum_{j=1}^{k} v^{j} \tilde{x}\left(\vec{e}_{j}\right) .
$$

If $k=2$, then equation (3) becomes

$$
x_{n}-a_{1} x_{n-1}-a_{2} x_{n-2}=0, \quad n \in \mathbb{N}_{0},
$$

where $a_{2} \neq 0$, and we have $\vec{e}_{1}=(1,0)$ and $\vec{e}_{2}=(0,1)$. Since

$$
f_{-1}=1, \quad f_{0}=0 \text { and } f_{1}=1,
$$

we see that the solution to equation (1) corresponding to the vector $\vec{e}_{1}$ is $\left(f_{n-1}\right)_{n \in \mathbb{N}_{0}}$, while the one corresponding to the vector $\vec{e}_{2}$ is $\left(f_{n}\right)_{n \in \mathbb{N}_{0}}$. Since every $\vec{v} \in \mathbb{R}^{2}$ has the form $\vec{v}=$ $v^{1} \vec{e}_{1}+v^{2} \vec{e}_{2}$, by using the relations in (4), we have

$$
\left(x_{n}(\vec{v})\right)_{n \in \mathbb{N}_{0}}=I_{2}(\vec{v})=v^{1} I_{2}\left(\vec{e}_{1}\right)+v^{2} I_{2}\left(\vec{e}_{2}\right)=v^{1}\left(f_{n-1}\right)_{n \in \mathbb{N}_{0}}+v^{2}\left(f_{n}\right)_{n \in \mathbb{N}_{0}} .
$$

From (7) with $n=0$ and $n=1$, it follows respectively that $x_{0}(\vec{v})=v^{1} f_{-1}+v^{2} f_{0}$ and $x_{1}(\vec{v})=v^{1} f_{0}+v^{2} f_{1}$, from which along with (6) it follows that $v^{1}=x_{0}(\vec{v})$ and $v^{2}=x_{1}(\vec{v})$. 
Hence

$$
x_{n}(\vec{v})=x_{0}(\vec{v}) f_{n-1}+x_{1}(\vec{v}) f_{n}, \quad n \in \mathbb{N}_{0},
$$

which is a known theoretical way how relation (2) is obtained.

Remark 1 Relation (2) can be also obtained in a quite simple elementary way by using the de Moivre formula (see, e.g., [30]).

Representation formula (2) suggests the following natural and interesting question.

Question Which homogeneous linear difference equations with constant coefficients have the general solution representation formula of the form in (2)?

Some recent representations of general solutions to some solvable difference equations and systems can be found, e.g., in [26-31, 33-36].

Here we give an answer to the question. Beside this we also explain an interesting elementary procedure for finding a representation of general solution to equation (3) in terms of the coefficients $a_{1}, a_{2}, \ldots, a_{k}$, initial values $x_{0}, x_{1}, \ldots, x_{k-1}$, and an extension of the Fibonacci sequence. The representation and some details should be folklore, but the whole procedure leading to it could be new and is interesting.

Before we state and prove the main results in this note, recall that since $a_{k} \neq 0$, every solution to equation (3) can be defined for all negative values of indices $n$, by using the following obvious consequence of the equation:

$$
x_{n-k}=\frac{x_{n}-\sum_{j=1}^{k-1} a_{j} x_{n-j}}{a_{k}} .
$$

For example, for $n=-1$, we get

$$
x_{-(k+1)}=\frac{x_{-1}-\sum_{j=1}^{k-1} a_{j} x_{-(j+1)}}{a_{k}},
$$

(this is, for example, used to get the value of $f_{-1}$ in (6)).

\section{Main results}

This section formulates and proves the main results in this paper. First, we give an answer to the above question.

\subsection{Answer to the question}

Now we turn to the above question when $k=2, a_{1} \in \mathbb{R}$, and $a_{2} \neq 0$, that is, to find all $a_{1}$ and $a_{2}$ such that the following representation holds:

$$
x_{n}(\vec{v})=x_{0}(\vec{v}) y_{n-1}\left(\vec{v}_{0}\right)+x_{1}(\vec{v}) y_{n}\left(\vec{v}_{0}\right), \quad n \in \mathbb{N}_{0},
$$

for some $\vec{v}_{0} \in \mathbb{R}^{2}$ and for every solution to equation (5), as well as to the corresponding question for the case of equation (3) for arbitrary $k \in \mathbb{N} \backslash\{1\}$.

The following theorem gives an answer to the question. 
Theorem 1 Consider equation (3) with $n \geq k \geq 2, a_{j} \in \mathbb{R}, j=\overline{1, k}$, and $a_{k} \neq 0$. Then there is a vector $\vec{v}_{0}=\left(v_{0}^{1}, \ldots, v_{0}^{k}\right) \in \mathbb{R}^{k}$ and $\tilde{y}\left(\vec{v}_{0}\right) \in \operatorname{Sol}\left(L_{k, \vec{a}}\right)$ such that

$$
y_{j}\left(\vec{v}_{0}\right)=v_{0}^{j+1}, \quad j=0, k-1,
$$

and that

$$
x_{n}(\vec{v})=x_{0}(\vec{v}) y_{n-1}\left(\vec{v}_{0}\right)+x_{1}(\vec{v}) y_{n}\left(\vec{v}_{0}\right)+\cdots+x_{k-1}(\vec{v}) y_{n+k-2}\left(\vec{v}_{0}\right)
$$

for every $n \in \mathbb{N}_{0}$, vector $\vec{v} \in \mathbb{R}^{k}$, and solution $\tilde{x}(\vec{v})$ to equation (3) if and only if $k=2, a_{2}=1$, and $\vec{v}_{0}=\left(v_{0}^{1}, v_{0}^{2}\right)=(0,1)$.

Proof Assume that representation (10) holds. Then for $n=0$ we have

$$
x_{0}(\vec{v})=x_{0}(\vec{v}) y_{-1}\left(\vec{v}_{0}\right)+x_{1}(\vec{v}) y_{0}\left(\vec{v}_{0}\right)+\cdots+x_{k-1}(\vec{v}) y_{k-2}\left(\vec{v}_{0}\right)
$$

for every $\vec{v} \in \mathbb{R}^{k}$, i.e., the identity

$$
\left(y_{-1}\left(\vec{v}_{0}\right)-1\right) x_{0}+y_{0}\left(\vec{v}_{0}\right) x_{1}+\cdots+y_{k-2}\left(\vec{v}_{0}\right) x_{k-1} \equiv 0
$$

must hold for every $\left(x_{0}, x_{1}, \ldots, x_{k-1}\right) \in \mathbb{R}^{k}$, from which it follows that

$$
y_{-1}\left(\vec{v}_{0}\right)=1, \quad y_{j}\left(\vec{v}_{0}\right)=0, \quad j=\overline{0, k-2} .
$$

From this and using the fact that $\tilde{y}\left(\vec{v}_{0}\right) \in \operatorname{Sol}\left(L_{k, \vec{a}}\right)$, we get

$$
y_{k-1}\left(\vec{v}_{0}\right)=\sum_{j=1}^{k} a_{j} y_{k-1-j}\left(\vec{v}_{0}\right)=a_{k} .
$$

For $n=1$ we have

$$
x_{1}(\vec{v})=x_{0}(\vec{v}) y_{0}\left(\vec{v}_{0}\right)+x_{1}(\vec{v}) y_{1}\left(\vec{v}_{0}\right)+\cdots+x_{k-2}(\vec{v}) y_{k-2}\left(\vec{v}_{0}\right)+x_{k-1}(\vec{v}) y_{k-1}\left(\vec{v}_{0}\right)
$$

for every $\vec{v} \in \mathbb{R}^{k}$. From (13)-(15), it follows that the identity

$$
x_{1}-a_{k} x_{k-1} \equiv 0
$$

must hold for every $x_{1}, x_{k-1} \in \mathbb{R}$, which is only possible if $k=2$ and $a_{k}=a_{2}=1$, from which along with (13) and (14) it follows that $0=y_{0}\left(\vec{v}_{0}\right)=v_{0}^{1}$ and $1=y_{1}\left(\vec{v}_{0}\right)=v_{0}^{2}$.

Now assume that $k=2, a_{2}=1$ and $\vec{v}_{0}=(0,1)$. Then (3) becomes

$$
x_{n}-a_{1} x_{n-1}-x_{n-2}=0, \quad n \geq 2 .
$$

An easy computation shows that

$$
y_{n}\left(\vec{v}_{0}\right)=\frac{\lambda_{1}^{n}-\lambda_{2}^{n}}{\lambda_{1}-\lambda_{2}}=\frac{\lambda_{1}^{n}-\lambda_{2}^{n}}{\sqrt{a_{1}^{2}+4}}
$$


where

$$
\lambda_{1}=\frac{a_{1}+\sqrt{a_{1}^{2}+4}}{2} \text { and } \lambda_{2}=\frac{a_{1}-\sqrt{a_{1}^{2}+4}}{2} .
$$

On the other hand, by calculating the solution to (17) with initial values $x_{0}(\vec{v})=v^{1}$ and $x_{1}(\vec{v})=v^{2}$, using (18) and Viete's formulas, we obtain

$$
\begin{aligned}
x_{n}(\vec{v}) & =\frac{\left(x_{0}(\vec{v}) \lambda_{2}-x_{1}(\vec{v})\right) \lambda_{1}^{n}+\left(x_{1}(\vec{v})-x_{0}(\vec{v}) \lambda_{1}\right) \lambda_{2}^{n}}{\lambda_{2}-\lambda_{1}} \\
& =-x_{0}(\vec{v}) \lambda_{1} \lambda_{2} \frac{\lambda_{1}^{n-1}-\lambda_{2}^{n-1}}{\lambda_{1}-\lambda_{2}}+x_{1}(\vec{v}) \frac{\lambda_{1}^{n}-\lambda_{2}^{n}}{\lambda_{1}-\lambda_{2}} \\
& =x_{0}(\vec{v}) y_{n-1}\left(\vec{v}_{0}\right)+x_{1}(\vec{v}) y_{n}\left(\vec{v}_{0}\right)
\end{aligned}
$$

for every $\vec{v}=\left(v^{1}, v^{2}\right)=\left(x_{0}(\vec{v}), x_{1}(\vec{v})\right)$, showing that representation (10) holds in this case, as desired.

\subsection{A representation of general solution to equation (3)}

Theorem 1 shows that for the case $k=2$ the solution to equation (3) satisfying the initial conditions $x_{0}=0$ and $x_{1}=1$ is of some representation importance for the general solution to the difference equation.

It is interesting to find the solution to equation (3) which naturally generalizes the Fibonacci sequence and at the same time plays the corresponding role in representation of general solution to the equation. Here we present an analysis which naturally leads to the solution. The analysis will show that the solution satisfies the initial conditions

$$
\begin{aligned}
& x_{j}=0, \quad j=\overline{0, k-2}, \\
& x_{k-1}=1 .
\end{aligned}
$$

The solution can be found also by using some other methods, but our aim is to get it in a direct and elementary way, that is, without using any non-elementary theorem.

To give an answer to the problem, we use here the procedure which some readers might have seen rather in linear algebra (in finding $n$th powers of matrices) than in dealing with real or complex numbers. This time we will apply it to the $n$th powers of numbers.

Since the roots

$$
\lambda_{1,2}=\frac{1 \pm \sqrt{5}}{2}
$$

of the characteristic polynomial

$$
P_{2}(\lambda)=\lambda^{2}-\lambda-1
$$

associated with difference equation (1) are different, here we assume that the zeros $\lambda_{j}$, $j=\overline{1, k}$, of the characteristic polynomial

$$
P_{k}(\lambda)=\lambda^{k}-\sum_{i=1}^{k} a_{i} \lambda^{k-i}
$$


associated with equation (3) are mutually different, that is, $\lambda_{i} \neq \lambda_{j}, i \neq j$, for $i, j \in$ $\{1,2, \ldots, k\}$.

In this case, general solution to equation (3) has the following form:

$$
x_{n}=\sum_{j=1}^{k} c_{j} \lambda_{j}^{n}, \quad n \in \mathbb{N}_{0},
$$

(the result essentially known to D. Bernoulli yet, [4]).

Note that

$$
\lambda_{j}^{k}=\sum_{i=1}^{k} a_{i} \lambda_{j}^{k-i}
$$

for each $j \in\{1,2, \ldots, k\}$.

By multiplying equality (23) by $\lambda_{j}$, then using again the equality and some simple calculations, we have

$$
\begin{aligned}
\lambda_{j}^{k+1} & =\sum_{i=1}^{k} a_{i} \lambda_{j}^{k-i+1}=a_{1} \lambda_{j}^{k}+\sum_{i=2}^{k} a_{i} \lambda_{j}^{k-i+1} \\
& =a_{1}\left(\sum_{i=1}^{k} a_{i} \lambda_{j}^{k-i}\right)+\sum_{i=1}^{k-1} a_{i+1} \lambda_{j}^{k-i} \\
& =a_{1} a_{k} \lambda_{j}^{0}+\sum_{i=1}^{k-1}\left(a_{1} a_{i}+a_{i+1}\right) \lambda_{j}^{k-i} \\
& =\sum_{i=1}^{k} a_{k+1}^{(1)} \lambda_{j}^{k-i}
\end{aligned}
$$

where

$$
a_{k+1}^{(i)}:=a_{1} a_{i}+a_{i+1}, \quad i=\overline{1, k-1}, \quad a_{k+1}^{(i)}:=a_{1} a_{k} .
$$

Hence, the quantity $\lambda_{j}^{k+1}$ has the same type of representation as $\lambda_{j}^{k}$, but with different coefficients, which are given by the relations in (25).

Assume that we have proved

$$
\lambda_{j}^{n}=\sum_{i=1}^{k} a_{n}^{(i)} \lambda_{j}^{k-i}
$$

for some $n \geq k+1$. 
Then, as above, by multiplying relation (26) by $\lambda_{j}$, suitable grouping of summands, and application of equality (23), after some simple calculations, it follows that

$$
\begin{aligned}
\lambda_{j}^{n+1} & =\sum_{i=1}^{k} a_{n}^{(i)} \lambda_{j}^{k-i+1}=a_{n}^{(1)} \lambda_{j}^{k}+\sum_{i=2}^{k} a_{n}^{(i)} \lambda_{j}^{k-i+1} \\
& =a_{n}^{(1)}\left(\sum_{i=1}^{k} a_{i} \lambda_{j}^{k-i}\right)+\sum_{i=1}^{k-1} a_{n}^{(i+1)} \lambda_{j}^{k-i} \\
& =a_{k} a_{n}^{(1)} \lambda_{j}^{0}+\sum_{i=1}^{k-1}\left(a_{i} a_{n}^{(1)}+a_{n}^{(i+1)}\right) \lambda_{j}^{k-i} \\
& =\sum_{i=1}^{k} a_{n+1}^{(i)} \lambda_{j}^{k-i}
\end{aligned}
$$

where

$$
\begin{aligned}
& a_{n+1}^{(i)}:=a_{i} a_{n}^{(1)}+a_{n}^{(i+1)}, \quad i=\overline{1, k-1}, \\
& a_{n+1}^{(k)}:=a_{k} a_{n}^{(1)} .
\end{aligned}
$$

From the relations in (23), (28), and the method of mathematical induction, it follows that relation (26) holds for every $n \geq k$, where the sequences $\left(a_{n}^{(i)}\right)_{n \geq 0}, i=\overline{1, k}$, satisfy the system of difference equations (28) and the initial conditions

$$
a_{k}^{(i)}=a_{i}, \quad i=\overline{1, k}
$$

To define the sequences $\left(a_{n}^{(i)}\right), i=\overline{1, k}$, for every $n \in \mathbb{N}_{0}$, we should choose the initial values in the following natural way:

$$
\lambda_{j}^{n}=\sum_{i=1}^{k} a_{n}^{(i)} \lambda_{j}^{k-i}
$$

for $n=\overline{0, k-1}$, so that

$$
a_{n}^{(i)}=0, \quad i \neq k-n, \quad a_{n}^{(i)}=1, \quad i=k-n .
$$

From (28) we have

$$
\begin{aligned}
a_{n}^{(1)}= & a_{1} a_{n-1}^{(1)}+a_{n-1}^{(2)} \\
= & a_{1} a_{n-1}^{(1)}+a_{2} a_{n-2}^{(1)}+a_{n-2}^{(3)} \\
& \cdots \\
= & a_{1} a_{n-1}^{(1)}+a_{2} a_{n-2}^{(1)}+\cdots+a_{k} a_{n-k}^{(1)}
\end{aligned}
$$

for $n \geq k$, while from (29) we have

$$
a_{n}^{(1)}=0, \quad n=\overline{0, k-2}, \quad a_{k-1}^{(1)}=1 .
$$


Hence, the sequence $\left(a_{n}^{(1)}\right)_{n \in \mathbb{N}_{0}}$ is the solution to difference equation (3) satisfying initial conditions (20), and this is one of the ways how the solution appears naturally.

From the relations in (28) we also have

$$
\begin{aligned}
a_{n}^{(i)}= & a_{i} a_{n-1}^{(1)}+a_{n-1}^{(i+1)} \\
= & a_{i} a_{n-1}^{(1)}+a_{i+1} a_{n-2}^{(1)}+a_{n-2}^{(i+2)} \\
& \cdots \\
= & a_{i} a_{n-1}^{(1)}+a_{i+1} a_{n-2}^{(1)}+\cdots+a_{k} a_{n-k+i-1}^{(1)} \\
= & \sum_{l=i}^{k} a_{l} a_{n-l+i-1}^{(1)}
\end{aligned}
$$

for $n \geq k$.

Employing relation (32) in (26), we obtain the following representation of the sequence $\left(\lambda_{j}^{n}\right)_{n \in \mathbb{N}_{0}}$ in terms of the powers $\lambda_{j}^{l}, l=\overline{0, k-1}$, coefficients of equation (3), and the sequence $\left(a_{n}^{(1)}\right)_{n \in \mathbb{N}_{0}}$ :

$$
\lambda_{j}^{n}=\sum_{i=1}^{k} \lambda_{j}^{k-i} \sum_{l=i}^{k} a_{l} a_{n-l+i-1}^{(1)}, \quad n \in \mathbb{N}_{0} .
$$

By changing the order of summation in formula (33) and using a change of variables of indices, we have

$$
\begin{aligned}
\lambda_{j}^{n} & =\sum_{i=1}^{k} \lambda_{j}^{k-i} \sum_{l=i}^{k} a_{l} a_{n-l+i-1}^{(1)}=\sum_{l=1}^{k} \sum_{i=1}^{l} \lambda_{j}^{k-i} a_{l} a_{n-l+i-1}^{(1)} \\
& =\sum_{s=1}^{k} a_{n-s}^{(1)} \sum_{t=1}^{k-s+1} \lambda_{j}^{k-t} a_{t+s-1} .
\end{aligned}
$$

Employing (34) in (22), it follows that the general solution to equation (3) in the case when all the roots $\lambda_{j}, j=\overline{1, k}$, of the characteristic polynomial associated with the equation are mutually different has the following representation:

$$
x_{n}=\sum_{j=1}^{k} c_{j} \sum_{s=1}^{k} a_{n-s}^{(1)} \sum_{t=1}^{k-s+1} \lambda_{j}^{k-t} a_{t+s-1}, \quad n \in \mathbb{N}_{0} .
$$

By changing the order of summation in (35), we have

$$
x_{n}=\sum_{s=1}^{k} a_{n-s}^{(1)} \sum_{t=1}^{k-s+1} a_{t+s-1} \sum_{j=1}^{k} c_{j} \lambda_{j}^{k-t}, \quad n \in \mathbb{N}_{0} .
$$

Now note that

$$
\sum_{j=1}^{k} c_{j} \lambda_{j}^{k-t}=x_{k-t}
$$

(see (22)) 
Combining (36) and (37), we have

$$
x_{n}=\sum_{s=1}^{k} a_{n-s}^{(1)} \sum_{t=1}^{k-s+1} a_{t+s-1} x_{k-t}, \quad n \in \mathbb{N}_{0} .
$$

By using the change of variables $j=k-t$, relation (38) can be written in the following way:

$$
x_{n}=\sum_{s=1}^{k} a_{n-s}^{(1)} \sum_{j=s-1}^{k-1} a_{k-j+s-1} x_{j}, \quad n \in \mathbb{N}_{0},
$$

which is a formula for general solution to equation (3) in terms of the roots $\lambda_{j}, j=\overline{1, k}$, of the characteristic polynomial associated with the equation, coefficients of the equation, and the sequence $a_{n}^{(1)}$.

By changing the order of summation in formula (39), we get

$$
x_{n}=\sum_{j=0}^{k-1} x_{j} \sum_{i=0}^{j} a_{k-j+i} a_{n-i-1}^{(1)}, \quad n \in \mathbb{N}_{0}
$$

or in the developed form, the following nice formula:

$$
x_{n}=x_{0}\left(a_{k} a_{n-1}^{(1)}\right)+x_{1}\left(a_{k-1} a_{n-1}^{(1)}+a_{k} a_{n-2}^{(1)}\right)+\cdots+x_{k-1}\left(a_{1} a_{n-1}^{(1)}+\cdots+a_{k} a_{n-k}^{(1)}\right)
$$

for $n \in \mathbb{N}_{0}$.

From the above consideration we see that we have given an interesting elementary proof of the following theorem, which should be folklore.

Theorem 2 Consider equation (3) with $n \geq k \geq 2, a_{j} \in \mathbb{R}, j=\overline{1, k}, a_{k} \neq 0$. Assume that the zeros $\lambda_{j}, j=\overline{1, k}$, of the characteristic polynomial associated with the equation are mutually different. If $\left(a_{n}^{(1)}\right)_{n \in \mathbb{N}_{0}}$ is the solution to the equation satisfying the initial conditions in (20), then general solution to the equation can be written in the form (39), as well as in the equivalent form (40).

Remark 2 In the case when all the zeros $\lambda_{j}, j=\overline{1, k}$, of the characteristic polynomial associated with equation (3) are mutually different, then the solution $\left(a_{n}^{(1)}\right)_{n \in \mathbb{N}_{0}}$ in Theorem 2 has the following nice closed form formula:

$$
a_{n}^{(1)}=\sum_{j=1}^{k} \frac{\lambda_{j}^{n}}{\prod_{i \neq j}\left(\lambda_{j}-\lambda_{i}\right)}=\sum_{j=1}^{k} \frac{\lambda_{j}^{n}}{P_{k}^{\prime}\left(\lambda_{j}\right)}, \quad n \in \mathbb{N}_{0} .
$$

Formula (41) follows from the following relations:

$$
\sum_{j=1}^{k} \frac{\lambda_{j}^{l}}{P_{k}^{\prime}\left(\lambda_{j}\right)}=0
$$

for $l=\overline{0, k-2}$, and

$$
\sum_{j=1}^{k} \frac{\lambda_{j}^{k-1}}{P_{k}^{\prime}\left(\lambda_{j}\right)}=1
$$


discovered by Lagrange (see, for example, [12], and also Lemma 1 in [35]), and the form of general solution to equation (3). We have used formula (41) recently in several papers on solvability of difference equations and systems of difference equations (see, e.g., [27$29,34-36])$.

By using (41) in (39), we also obtain the following representation of general solution to equation (3) for the case when the zeros $\lambda_{j}, j=\overline{1, k}$, of the characteristic polynomial associated with the equation are mutually different:

$$
x_{n}=\sum_{s=1}^{k} \sum_{i=1}^{k} \frac{\lambda_{i}^{n-s}}{P_{k}^{\prime}\left(\lambda_{i}\right)} \sum_{j=s-1}^{k-1} a_{k-j+s-1} x_{j}, \quad n \in \mathbb{N}_{0} .
$$

Remark 3 From the representation in (40) we obtain another proof of Theorem 1 for the case when $\vec{v}_{0}=(0, \ldots, 0,1)$. Namely, the coefficient at $x_{0}$ in (40) is equal to $a_{k} a_{n-1}^{(1)}$, from which it follows that $a_{k}$ must be equal to one, whereas the coefficient at $x_{k-1}$ in (40) is equal to $a_{1} a_{n-1}^{(1)}+\cdots+a_{k} a_{n-k}^{(1)}$, which by the definition must be $a_{n}^{(1)}$, from which together with the form of solution representation in (10) it follows that delay $k$ must be equal to two.

Remark 4 If $k=2$, then from (40) we have

$$
x_{n}=x_{0} a_{2} a_{n-1}^{(1)}+x_{1} a_{n}^{(1)}, \quad n \in \mathbb{N}_{0},
$$

a formula which is a consequence of the de Moivre formula for the second-order difference equation. A detailed explanation for representation (42) can be found, for example, in [26]. We would also like to say that the formula holds also for the case when both roots of the characteristic polynomial associated with the equation are equal (see [26]).

Remark 5 Another representation for general solution to equation (3) can be obtained by calculating the coefficients $c_{j}, j=\overline{1, k}$, in formula (22) in terms of the initial values $x_{0}, x_{1}, \ldots, x_{k-1}$, and the roots $\lambda_{j}, j=\overline{1, k}$, of the characteristic polynomial. Namely, it must be

$$
\begin{aligned}
& c_{1}+c_{2}+\cdots+c_{k}=x_{0} \\
& \vdots \\
& c_{1} \lambda_{1}^{j}+c_{2} \lambda_{2}^{j}+\cdots+c_{k} \lambda_{k}^{j}=x_{j} \\
& \vdots \\
& c_{1} \lambda_{1}^{k-1}+c_{2} \lambda_{2}^{k-1}+\cdots+c_{k} \lambda_{k}^{k-1}=x_{k-1},
\end{aligned}
$$


from which it follows that

$$
\begin{aligned}
c_{i} & =\left|\begin{array}{ccccccc}
1 & \ldots & 1 & x_{0} & 1 & \ldots & 1 \\
\vdots & \vdots & \vdots & \vdots & \vdots & \vdots & \vdots \\
\lambda_{1}^{j} & \ldots & \lambda_{i-1}^{j} & x_{j} & \lambda_{i+1}^{j} & \ldots & \lambda_{k}^{j} \\
\vdots & \vdots & \vdots & \vdots & \vdots & \vdots & \vdots \\
\lambda_{1}^{k-1} & \ldots & \lambda_{i-1}^{k-1} & x_{k-1} & \lambda_{i+1}^{k-1} & \ldots & \lambda_{k}^{k-1}
\end{array}\right| \cdot\left|\begin{array}{cccc}
1 & 1 & \ldots & 1 \\
\vdots & \vdots & \vdots & \vdots \\
\lambda_{1}^{j} & \lambda_{2}^{j} & \ldots & \lambda_{k}^{j} \\
\vdots & \vdots & \vdots & \vdots \\
\lambda_{1}^{k-1} & \lambda_{2}^{k-1} & \ldots & \lambda_{k}^{k-1}
\end{array}\right|^{-1} \\
& =\sum_{j=0}^{k-1} x_{j}(-1)^{i+j+1} \frac{W_{j+1, i}}{V_{k}\left(\lambda_{1}, \lambda_{2}, \ldots, \lambda_{k}\right)}
\end{aligned}
$$

for $i=\overline{1, k}$, where $V_{k}\left(\lambda_{1}, \lambda_{2}, \ldots, \lambda_{k}\right)$ is the so-called Vandermonde determinant, whereas $W_{j+1, i}$ is the minor corresponding to the element $x_{j}$ in the first determinant in (43) (i.e., to the element at the position $(j+1, i))$. Many determinants and methods for calculating them can be found, e.g., in [15] and [23].

By calculating the determinants in (43), and consequently obtaining in this way some formulas for constants $c_{i}, i=\overline{1, k}$, in terms of the initial values $x_{0}, x_{1}, \ldots, x_{k-1}$ and characteristic values $\lambda_{j}, j=\overline{1, k}$, and using such obtained formulas for $c_{i}$ s in (22), after some further calculations and algebraic manipulations, we can also obtain the representation in Theorem 2, but this time in a more complicated way. It should be also pointed out that, if this method is used, then the solution $\left(a_{n}^{(1)}\right)_{n \in \mathbb{N}_{0}}$ to equation (3) does not appear so directly as it was the case in the analysis preceding formulation of Theorem 2.

Bearing in mind Remark 4, it is natural to expect that formula (40) also holds in the case when some of the roots of the characteristic polynomial (21) are equal. Since (40) is a solution to equation (3) (note that it is a linear combination of solutions $a_{n-s}^{(1)}$ ), to prove the claim it is enough to check that its initial values in all possible cases are $x_{0}, x_{1}, \ldots, x_{k-1}$.

Let $n=0$. Then, by using the relation

$$
a_{k-j}^{(1)}=a_{1} a_{k-j-1}^{(1)}+\cdots+a_{k} a_{-j}^{(1)},
$$

$j=\overline{1, k}$, as well as (31), we have

$$
\begin{aligned}
& x_{0}\left(a_{k} a_{-1}^{(1)}\right)+x_{1}\left(a_{k-1} a_{-1}^{(1)}+a_{k} a_{-2}^{(1)}\right)+\cdots+x_{k-1}\left(a_{1} a_{-1}^{(1)}+\cdots+a_{k} a_{-k}^{(1)}\right) \\
& \quad=x_{0}\left(a_{k-1}^{(1)}-\sum_{j=1}^{k-1} a_{j} a_{k-j-1}^{(1)}\right)+x_{1}\left(a_{k-2}^{(1)}-\sum_{j=1}^{k-2} a_{j} a_{k-j-2}^{(1)}\right)+\cdots+x_{k-1} a_{0}^{(1)} \\
& \quad=x_{0} a_{k-1}^{(1)}=x_{0},
\end{aligned}
$$

so the claim holds in this case. 
If $n=i \in\{1,2, \ldots, k-1\}$, then the calculations are a bit more complicated, but still quite simple. We have

$$
\begin{aligned}
& x_{0}\left(a_{k} a_{i-1}^{(1)}\right)+\cdots+x_{i}\left(a_{k-i} a_{i-1}^{(1)}+\cdots+a_{k} a_{-1}^{(1)}\right)+x_{i+1}\left(a_{k-i-1} a_{i-1}^{(1)}+\cdots+a_{k} a_{-2}^{(1)}\right) \\
& +\cdots+x_{k-1}\left(a_{1} a_{i-1}^{(1)}+\cdots+a_{k} a_{i-k}^{(1)}\right) \\
& \quad=x_{i} a_{k} a_{-1}^{(1)}+x_{i+1}\left(a_{k-2}^{(1)}-\sum_{j=1}^{k-i-2} a_{j} a_{k-j-2}^{(1)}\right)+\cdots+x_{k-1} a_{i}^{(1)}=x_{i} a_{k} a_{-1}^{(1)}=x_{i}
\end{aligned}
$$

since

$$
a_{k} a_{-1}^{(1)}=a_{k-1}^{(1)}-a_{1} a_{k-2}^{(1)}-\cdots-a_{k-1} a_{0}^{(1)}=a_{k-1}^{(1)}=1 .
$$

From this we see that the following extension of Theorem 2 holds.

Theorem 3 Consider equation (3) with $n \geq k \geq 2, a_{j} \in \mathbb{R}, j=\overline{1, k}, a_{k} \neq 0$. If $\left(a_{n}^{(1)}\right)_{n \in \mathbb{N}_{0}}$ is the solution to the equation satisfying the initial conditions in (20), then general solution to the equation can be written in the form (39), as well as in the equivalent form (40).

\section{Acknowledgements}

The work of Zdeněk Šmarda was supported by the project FEKT-S-20-6225 of Brno University of Technology.

Funding

Brno University of Technology, project FEKT-S-20-6225.

\section{Availability of data and materials}

Not applicable.

\section{Competing interests}

The authors declare that they have no competing interests.

\section{Authors' contributions}

The authors have contributed equally to the writing of this paper. They read and approved the manuscript.

\section{Author details}

${ }^{1}$ Mathematical Institute of the Serbian Academy of Sciences, Knez Mihailova 36/III, 11000 Beograd, Serbia. ${ }^{2}$ Department of Medical Research, China Medical University Hospital, China Medical University, Taichung 40402, Taiwan, Republic of China. ${ }^{3}$ Brno University of Technology, Faculty of Electrical Engineering and Communication, Department of Mathematics, Technicka 3058/10, CZ - 61600 Brno, Czech Republic. ${ }^{4}$ Faculty of Electrical Engineering, Belgrade University, Bulevar Kralja Aleksandra 73, 11000 Beograd, Serbia. ${ }^{5}$ Faculty of Mechanical and Civil Engineering in Kraljevo, University of Kragujevac, Kraljevo, Serbia. ${ }^{6}$ Department of Mathematical Sciences, Appalachian State University, Boone, NC 28608, USA.

\section{Publisher's Note}

Springer Nature remains neutral with regard to jurisdictional claims in published maps and institutional affiliations.

Received: 18 August 2020 Accepted: 2 September 2020 Published online: 10 September 2020

\section{References}

1. Alfred, B.U.: An Introduction to Fibonacci Discovery. The Fibonacci Association (1965)

2. Berezansky, L., Braverman, E.: On impulsive Beverton-Holt difference equations and their applications. J. Differ. Equ. Appl. 10(9), 851-868 (2004)

3. Berg, L., Stević, S.: On some systems of difference equations. Appl. Math. Comput. 218, 1713-1718 (2011)

4. Bernoulli, D.: Observationes de seriebus quae formantur ex additione vel substractione quacunque terminorum se mutuo consequentium, ubi praesertim earundem insignis usus pro inveniendis radicum omnium aequationum algebraicarum ostenditur. Commentarii Acad. Petropol. III 1728, 85-100 (1732) (in Latin)

5. Boole, G.: A Treatsie on the Calculus of Finite Differences, 3rd edn. Macmillan \& Co., London (1880)

6. de Moivre, A.: De fractionibus algebraicis radicalitate immunibus ad fractiones simpliciores reducendis, deque summandis terminis quarumdam serierum aequali intervallo a se distantibus. Philosophical Transactions 32, 162-178 (1722) (in Latin) 
7. de Moivre, A.: Miscellanea Analytica de Seriebus et Quadraturis. J. Tonson \& J. Watts, London (1730) (in Latin)

8. de Moivre, A.: The Doctrine of Chances, 3rd edn. Strand Publishing, London (1756)

9. Euler, L.: Introductio in Analysin Infinitorum, Tomus Primus (1748) Lausannae (in Latin)

10. Fort, T.: Finite Differences and Difference Equations in the Real Domain. Oxford Univ. Press, London (1948)

11. Jordan, C.: Calculus of Finite Differences. Chelsea, New York (1956)

12. Krechmar, V.A.: A Problem Book in Algebra. Mir, Moscow (1974) (original Russian version 1937)

13. Markoff, A.A.: Differenzenrechnung. Teubner, Leipzig (1896) (in German)

14. Milne-Thomson, L.M.: The Calculus of Finite Differences. Macmillan \& Co., London (1933)

15. Mitrinović, D.S.: Matrice i Determinante. Naučna Knjiga, Beograd (1989) (in Serbian)

16. Mitrinović, D.S., Adamović, D.D.: Nizovi i Redovi. Naučna Knjiga, Beograd (1980) (in Serbian)

17. Mitrinović, D.S., Kečkić, J.D.: Metodi Izračunavanja Konačnih Zbirova. Naučna Knjiga, Beograd (1984) (in Serbian)

18. Nörlund, N.E.: Vorlesungen über Differenzenrechnung. Springer, Berlin (1924) (in German)

19. Papaschinopoulos, G., Schinas, C.J.: On the behavior of the solutions of a system of two nonlinear difference equations. Commun. Appl. Nonlinear Anal. 5(2), 47-59 (1998)

20. Papaschinopoulos, G., Schinas, C.J.: Invariants for systems of two nonlinear difference equations. Differ. Equ. Dyn. Syst. 7, 181-196 (1999)

21. Papaschinopoulos, G., Schinas, C.J.: Invariants and oscillation for systems of two nonlinear difference equations. Nonlinear Anal., Theory Methods Appl. 46, 967-978 (2001)

22. Papaschinopoulos, G., Stefanidou, G.: Asymptotic behavior of the solutions of a class of rational difference equations. Int. J. Difference Equ. 5(2), 233-249 (2010)

23. Proskuryakov, I.V.: Sbornik Zadach po Lineynoy Algebre. Nauka, Moscow (1984) (in Russian)

24. Schinas, C.: Invariants for difference equations and systems of difference equations of rational form. J. Math. Anal. Appl. 216, 164-179 (1997)

25. Schinas, C.: Invariants for some difference equations. J. Math. Anal. Appl. 212, 281-291 (1997)

26. Stević, S.: Representation of solutions of bilinear difference equations in terms of generalized Fibonacci sequences. Electron. J. Qual. Theory Differ. Equ. 2014, Article ID 67 (2014)

27. Stević, S.: Solvability of the class of two-dimensional product-type systems of difference equations of delay-type (1, 3,1, 1). Symmetry 9, Article ID 200 (2017)

28. Stević, S.: Solvable product-type system of difference equations whose associated polynomial is of the fourth order. Electron. J. Qual. Theory Differ. Equ. 2017, Article ID 13 (2017)

29. Stević, S.: Solvable product-type system of difference equations with two dependent variables. Adv. Differ. Equ. 2017, Article ID 245 (2017)

30. Stević, S.: Representations of solutions to linear and bilinear difference equations and systems of bilinear difference equations. Adv. Differ. Equ. 2018, Article ID 474 (2018)

31. Stević, S.: General solutions to four classes of nonlinear difference equations and some of their representations. Electron. J. Qual. Theory Differ. Equ. 2019, Article ID 75 (2019)

32. Stević, S.: Solvability of some classes of nonlinear first-order difference equations by invariants and generalized invariants. Electron. J. Qual. Theory Differ. Equ. 2019, Article ID 36 (2019)

33. Stević, S., Iričanin, B., Kosmala, W., Šmarda, Z:: Note on the bilinear difference equation with a delay. Math. Methods Appl. Sci. 41, 9349-9360 (2018)

34. Stević, S., Iričanin, B., Kosmala, W., Šmarda, Z.: Representation of solutions of a solvable nonlinear difference equation of second order. Electron. J. Qual. Theory Differ. Equ. 2018, Article ID 95 (2018)

35. Stević, S., Iričanin, B., Šmarda, Z.: On a product-type system of difference equations of second order solvable in closed form. J. Inequal. Appl. 2015, Article ID 327 (2015)

36. Stević, S., Iričanin, B., Šmarda, Z:: On a solvable class of product-type systems of difference equations. Filomat 31(19), 6113-6129 (2017)

37. Vorobiev, N.V:: Fibonacci Numbers. Birkhäuser, Basel (2002)

\section{Submit your manuscript to a SpringerOpen ${ }^{\circ}$ journal and benefit from:}

- Convenient online submission

- Rigorous peer review

- Open access: articles freely available online

- High visibility within the field

- Retaining the copyright to your article

Submit your next manuscript at $\boldsymbol{\nabla}$ springeropen.com 